\title{
Mesenchymal stem cells seem too good to believe!
}

\author{
Friedrich C. Luft
}

Published online: 13 September 2014

(C) Springer-Verlag Berlin Heidelberg 2014

Mesenchymal stem cells (MSCs) are multipotent stromal cells that can differentiate into various cell types including osteoblasts, chondroblasts, adipocytes, vascular smooth muscle cells, and other cell types (Fig. 1). MSCs harbor unique immunomodulatory properties and therefore hold great promise in tissue engineering, since they not only directly participate in tissue regeneration and repair but also modulate the host's foreign body responses [1]. The signaling pathways regulating MSCs include bone morphogenetic proteins (BMPs), epidermal growth factors (EGF), transforming growth factors (TGF), wingless integration site (wnt) proteins, fibroblastic growth factor (FGF), and transcription factors, and have been reviewed earlier [2]. MSCs can maintain antiinflammatory, anti-fibrotic, antimicrobial, and regenerative properties, so that they could conceivably improve outcomes in various conditions featuring damaged tissues and inflammation [3]. The putative beneficial effects of MSCs include increased cell survival and proliferation, decreased inflammation, and suppression of immune function. With such a broad magical repertoire of effects, how could they miss? Failures have hardly been reported.

Could MSCs lower blood pressure or ameliorate hypertension-induced target-organ damage? MSCs have been injected into stroke-prone spontaneously hypertensive rats (SHR). The authors of that report claimed that MSCs had antioxidant and anti-apoptotic effects as well as the ability to repair hippocampal damage in SHR [4]. However, a reduction in blood pressure was not reported. Other investigators injected MSCs into tail veins of rats subjected to 2-kidney, 1-clip renovascular hypertension [5]. MSCs prevented the progressive increase in blood pressure, ameliorated target-

F. C. Luft $(\bowtie)$

Experimental and Clinical Research Center, Charité Medical Faculty,

Berlin, Germany

e-mail: luft@charite.de organ damage, reduced fibrosis, proteinuria, and inflammatory cytokines, suppressed the intrarenal renin-angiotensin system, decreased sympathetic hyperactivity, and infiltrated not only the kidneys but also the central nervous system in the rats. These are remarkable claims indeed!

Investigators have also applied MSCs to models of renal disease [6, 7]. In this issue of J Mol Med, Hu and colleagues treated Dahl salt-sensitive (S) rats with intrarenal MSCs [8]. Immunohistochemistry and flow-cytometry analyses showed a significantly reduced number of stem cell marker CD133+ cells in the renal medulla of Dahl S rats, compared to controls, suggesting a renal stem-cell deficiency. Rat MSCs or control cells were transplanted into the renal medulla in uninephrectomized Dahl S rats. High-salt diet resulted in sodium retention and hypertension in control Dahl $\mathrm{S}$ rats. These effects were significantly attenuated in MSC-treated

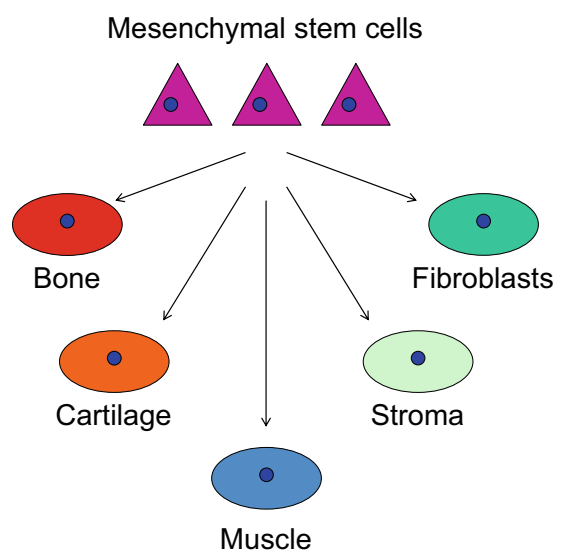

Fig. 1 Mesenchymal stem cells (MSCs) can give rise to many cell types such as adipocytes, cartilage, bone, tendon and ligaments, muscle, skin, and nerves. MSCs were initially isolated from bone marrow samples, but more recently, they have been found in many other types of tissue, such as simple skin and fat biopsies, resulting in a heterogeneous and abundant population of cells with high differentiation potential. MSCs are currently being investigated in various clinical trials 
rats. Moreover, high-salt diet-induced increases in proinflammatory monocyte chemoattractant protein-1 and interleukin$1 \beta$ were reduced in the renal medulla with MSCs. Immunostaining showed that high-salt diet-induced immune cell infiltration into the renal medulla was significantly inhibited by MSC treatment as well. These results suggested that a stemcell defect in the renal medulla could contribute to the hypertension in Dahl S rats and that MSC treatment attenuates the defect by an anti-inflammatory action. The authors relied on a commercial source for their rat MSCs (Texas A\&M Health Science). They used Brown-Norway control rats, since these are the reference strain in molecular genetic studies. The rats were given $2 \%$ saline (about similar to sea water) or a highsalt diet $8 \%$ by weight. To the authors' credit, blood pressure was measured by radio-telemetry.

Cowley et al. first drew attention to the renal medulla as a mechanistic focal point of salt-sensitive hypertension [9]. They implanted optical fibers to measure cortical and medullary blood flow and delivered vasoactive compounds into the medullary interstitial space. They tested nitric oxide, atrial natriuretic peptides, kinins, eicosanoids, vasopressin, and the effects of sympathetic nerves. Cowley et al. used long-term medullary interstitial captopril infusions that resulted in a lowering of arterial pressure [9]. The authors maintained that although blood flow to the inner renal medulla comprises less than $1 \%$ of the total renal blood flow, changes in flow to this region have a major effect on salt homeostasis and blood pressure.

These earlier studies certainly help the data presentation of $\mathrm{Hu}$ et al. [8]. However, not referenced or discussed in the current report is another important contribution from the Cowley laboratory. Feng et al. reported that the expression of a particular nicotinamide adenine dinucleotide phosphate (NADPH) oxidase subunit, p67(phox), was increased in response to a high-salt diet in the renal medulla of the Dahl S rat [10]. The authors found a mutation in the promoter of the gene encoding p67(phox) that contributed to increased NADPH activity. Feng et al. then "knocked out" p67(phox) in Dahl S rats using zinc-finger nucleases. These "treated" rats exhibited less salt-sensitive hypertension and renal medullary inflammation.

Missing in the current report is a strategy to reconcile these findings. Was the p67(phox) mutation described by Feng et al. [10] present in the authors' strain? Would a positive-control experiment be possible? For instance, could $\mathrm{Hu}$ et al. [8] deplete renal stem cells from the medulla of Brown-Norway control rats and produce the salt-sensitive Dahl S phenotype? Finally, what is it about these MSCs? What cell types did they generate and how were the effects mediated? Not all the recent news on stem cells has been good [11]. The findings will need to be replicated.

Respectfully,

Friedrich C. Luft

\section{References}

1. Hanson S, D'Souza RN, Hematti P (2014) Biomaterial-mesenchymal stem cell constructs for immunomodulation in composite tissue engineering. Tissue Eng A 20:2162-2168

2. Bhaskar B, Mekala NK, Rao PS, Baadhe RR (2014) Role of signaling pathways in mesenchymal stem cell differentiation. Curr Stem Cell Res Ther

3. Sutton MT, Bonfield TL (2014) Stem cells: innovations in clinical applications. Stem Cells Int 2014:516278

4. Calio ML, Marinho DS, Ko GM, Ribeiro RR, Carbonel AF, Oyama LM, Ormanji M, Guirao TP, Calio PL, Reis LA et al (2014) Transplantation of bone marrow mesenchymal stem cells decreases oxidative stress, apoptosis, and hippocampal damage in brain of a spontaneous stroke model. Free Radic Biol Med 70:141-154

5. Oliveira-Sales EB, Maquigussa E, Semedo P, Pereira LG, Ferreira VM, Camara NO, Bergamaschi CT, Campos RR, Boim MA (2013) Mesenchymal stem cells (msc) prevented the progression of renovascular hypertension, improved renal function and architecture. PLoS One 8:e78464

6. Dziedzic K, Pleniceanu O, Dekel B (2014) Kidney stem cells in development, regeneration and cancer. Sem Cell Develop Biol

7. Rosenberg ME (2013) Cell-based therapies in kidney disease. Kidney Int Suppl 3:364-367

8. Hu J, Zhu Q, Xia M, Guo TL, Wang Z, Li PL, Han WQ, Yi F, Li N (2014) Transplantation of mesenchymal stem cells into the renal medulla attenuated salt-sensitive hypertension in dahl s rat. J Mol Med. doi:10.1007/s00109-014-1199-1

9. Cowley AW Jr, Mattson DL, Lu S, Roman RJ (1995) The renal medulla and hypertension. Hypertension 25:663-673

10. Feng D, Yang C, Geurts AM, Kurth T, Liang M, Lazar J, Mattson DL, O'Connor PM, Cowley AW Jr (2012) Increased expression of nad(p)h oxidase subunit p67(phox) in the renal medulla contributes to excess oxidative stress and salt-sensitive hypertension. Cell Metab 15:201-208

11. Nowbar AN, Mielewczik M, Karavassilis M, Dehbi HM, Shun-Shin MJ, Jones S, Howard JP, Cole GD, Francis DP, Group Dw (2014) Discrepancies in autologous bone marrow stem cell trials and enhancement of ejection fraction (damascene): weighted regression and meta-analysis. BMJ 348:g2688 